\title{
White opaque substance visualized by magnifying narrow-band imaging is associated with intragastric acid conditions
}

\section{(ㄷ)(1) $\odot$}

Authors

Kazumi Togo' ${ }^{1}$, Tetsuya Ueo ${ }^{1}$, Kenshi Yao ${ }^{2}$, Kurato Wada', Hideho Honda ${ }^{1}$, Syotaro Inoue ${ }^{1}$, Masahide Fukuda', Yuka Yanai $^{1}$, Hirotoshi Yonemasu ${ }^{3}$, Kazunari Murakami ${ }^{4}$

Institutions

1 Department of Gastroenterology, Oita Red Cross Hospital, Japan

2 Department of Endoscopy, Fukuoka University Chikushi Hospital, Japan

3 Department of Pathology, Oita Red Cross Hospital, Japan

4 Department of Gastroenterology, Faculty of Medicine, Oita University, Japan

submitted 27.11.2017

accepted after revision 28.3.2018

Bibliography

DOI https://doi.org/10.1055/a-0611-4825 |

Endoscopy International Open 2018; 06: E830-E837

(C) Georg Thieme Verlag KG Stuttgart · New York

ISSN 2364-3722

Corresponding author

Tetsuya Ueo, MD, PhD, Department of Gastroenterology, Oita Red Cross Hospital, Chiyo-machi, Oita 870-0033, Japan Fax: +81-97-533-1207

ueo14@athena.ocn.ne.jp

\section{ABSTRACT}

Background and study aims The presence of white opaque substance (WOS) is an endoscopic marker of intestinal metaplasia. Considering that the nature of WOS is absorbed lipid droplets, lipase plays an important role in the lipid absorption process and is inactivated at strong acidity. WOS may only be present in a hypochlorhydria state following Helicobacter pylori infection, and, thus, may not be highly sensitive marker, especially in $\mathrm{H}$. pylori-eradicated patients. We investigated the relationship between WOS and gastric acid conditions.

Patients and methods A total of 501 patients were retrospectively evaluated for the presence of WOS at 2 regions of interest using magnifying narrow-band imaging. The $\mathrm{pH}$ level of collected gastric juice was also measured. Study end points were (1) prevalence of WOS and its relationship with gastric juice $\mathrm{pH}$ in 3 groups: $\mathrm{H}$. pylori-uninfected, $H$. pylori-infected, and $\mathrm{H}$. pylori-eradicated; (2) the relationship between prevalence of WOS and gastric juice $\mathrm{pH}$ before and after proton pump inhibitor (PPI) administration in 29 H. pylori-eradicated cases.

Results Prevalence of WOS was $0 \%$ (0/206), 28.4\% (31/ $109)$, and $3.2 \%(6 / 186)$ in the $H$. pylori-uninfected, H. pylori-infected, and $H$. pylori-eradicated groups, respectively. Mean gastric juice $\mathrm{pH}$ was significantly higher in WOS-positive cases than in WOS-negative cases in the H. pylori-infected and $H$. pylori-eradicated groups $(P<0.0001)$. Mean gastric juice $\mathrm{pH}$ increased from 1.1 to 6.9 after PPI administration and WOS prevalence increased from $0 \%$ (0/29) to $45 \%$ $(13 / 29)$ of cases.

Conclusion The prevalence of WOS is closely associated with the neutralization of intragastric $\mathrm{pH}$.

\section{Introduction}

Helicobacter pylori, when it infects the gastric mucosa, has been recognized as a major pathogen in the development of gastric cancer [1]. In particular, the presence of gastric intestinal metaplasia (IM) is thought to be an important risk in the development of gastric cancer because of its strong association with intestinal-type gastric cancer [2,3]. Appropriate surveillance of gastric IM could lead to earlier detection and treatment of gastric cancer, potentially leading to more favorable patient survival outcomes [4]. As gastric IM shows heterogeneous patchy distribution and is not distinctly visible by conventional white-light endoscopy alone, the evaluation of gastric IM is of limited accuracy. In the Kyoto global consensus report on $\mathrm{H}$. pylori gastritis, expert panels strongly recommend modern image-enhanced endoscopy, such as narrow-band imaging (NBI), for the accurate diagnosis of gastric IM [5].

Using magnifying $\mathrm{NBI}(\mathrm{M}-\mathrm{NBI})$, light blue crest (LBC) has been widely accepted as an endoscopic marker of gastric IM [6]. LBC is defined as a fine, blue-white line on the crests of the 
epithelial surface/gyri observable with M-NBI and histologically indicates the brush border of IM [6]. However, in a recent study, white opaque substance (WOS) observed with M-NBI was reported as another, novel, endoscopic marker of IM [7]. WOS on M-NBI was first reported by Yao et al. [8] as a substance present in the superficial part of gastric neoplasias that obscures the subepithelial microvascular architecture. According to fat (oilred $\mathrm{O}$ ) staining and immunohistochemical and immunoelectron microscopic studies for adipophilin, WOS in gastric epithelial neoplasia is an optical phenomenon caused by accumulated lipid droplets $[9,10]$. Studies of $H$. pylori infection-associated IM of the stomach using lipid staining and light microscopy or electron microscopy showed that the epithelium in IM has the ability to absorb lipid droplets [11,12].

Lipase plays a crucial role in the lipid digestion and absorption process and is known to be inactivated in strong acidity [13]. Therefore, we assume that the presence of absorbed lipid droplets as WOS in IM may represent a hypochlorhydria state caused by atrophic gastritis due to $H$. pylori infection and may not be a consistent phenomenon because it is influenced by acid recovery in $H$. pylori-eradicated patients. In other words, WOS may not be sufficiently sensitive as an endoscopic marker of IM in patients with recovered acid secretion after $H$. pylori eradication, an issue of critical importance that requires resolution. The Japanese government approved eradication treatment for patients with $H$. pylori-associated chronic gastritis under the National Health Insurance System from February 2013, and the number of patients who have received $H$. pylori eradication therapy is increasing in Japan. Moreover, the incidence of gastric cancer after $H$. pylori eradication has been increasing, especially in patients who have had observed IM in background mucosa [14]. Using routine screening endoscopy, accurate diagnosis of IM as a precancerous lesion in patients is required, including in those who have had $H$. pylori eradicated.

In this study, we aimed to clarify the clinical significance of the presence of WOS and its limitations in the changing gastric acid conditions in various chronic gastritis states secondary to H. pylori infection.

\section{Methods}

\section{Study design and participants}

This study was approved by the institutional review board of Oita Red Cross Hospital (IRB no. 190) and conducted according to the Declaration of Helsinki. All patients provided their written informed consent.

We retrospectively extracted 582 patients who underwent both upper gastrointestinal (GI) screening endoscopy with magnifying $\mathrm{NBI}$ and intragastric $\mathrm{pH}$ assessment at Oita Red Cross Hospital between April 2014 and July 2016. The data of fasting gastric juice used in this study were originally collected in our previous study on investigation of endoscopic findings that can predict gastric acid condition [15].

Subjects were excluded if they had following exclusion criteria: (1) patients who had a history of gastric resection; (2) with severe organ failure; (3) current intake of antiacid agents (within $5 \mathrm{~d}$ prior to the endoscopic examination); (4) patients within 6 mo of prior $H$. pylori eradication; (5) patients with an unclear $H$. pylori status or suggestive of spontaneous $H$. pylori eradication and; (6) endoscopic images insufficient for assessment. After 81 patients were excluded, a total of 501 patients were included in this study.

\section{Endoscopic procedure and $\mathrm{pH}$ measurement of fasting gastric juice}

A high-resolution magnifying upper GI endoscope (GIF-Q240Z; Olympus, Tokyo, Japan) or a high-definition magnifying upper Gl endoscope (GIF-H260Z, Olympus) and an electronic endoscopy system (EVIS LUCERA Spectrum, Olympus) were used. A soft black hood (MAJ-1989 for the GIF-Q240Z, MAJ-1990 for the GIF-H260Z; Olympus) was mounted at the tip of the endoscope to enable the endoscopist to fix a consistent focal distance between the tip of the endoscope and the gastric mucosa. Measurement of the $\mathrm{pH}$ of fasting gastric juice and M-NBI examinations were carried out by endoscopists K.T., T.U., K.W., H.H., S.I., M. F., and Y.Y.

Endoscopic study was performed following an overnight fast of $12 \mathrm{~h}$ or more. To avoid contamination affecting the accuracy of $\mathrm{pH}$ measurements, premedication (such as mucolytic or deforming agents) was not administered, except for local pharyngeal anesthesia with $8 \%$ lidocaine pump spray. Following endoscope insertion into the stomach, gastric juice pooled in the stomach was aspirated and collected endoscopically according to a previously described technique [16]. The $\mathrm{pH}$ of the collected sample was immediately measured using a 14-step $\mathrm{pH}$ test strip (pH 1-14; AS ONE Co., Osaka, Japan). Following this, the observer evaluated the presence or absence of WOS at 2 regions of interest using M-NBI. The observer then recorded the results on a digital filing system (NEXUS; Fujifilm Medical Co., Tokyo, Japan), and the images were subsequently doublechecked by an experienced M-NBI endoscopist (T.U.).

\section{Definitions of WOS}

The 2 regions of interest identified for assessment of presence of WOS were a $2 \times 2 \mathrm{~cm}$ area at the lesser curvature of the antrum, $2 \mathrm{~cm}$ oral to the pylorus ring, and a $2 \times 2 \mathrm{~cm}$ area at the lesser curvature of the gastric corpus, $4 \mathrm{~cm}$ oral to the angulus according to a previous report [17].

We defined the region of interest as WOS-positive when MNBI findings showed a white substance obscuring the subepithelial capillaries of the intervening parts between crypt openings regardless of the degree or distribution and regardless of LBC coexistence ( $\triangleright$ Fig. 1 a). A normal mucosa or LBC-positive but WOS-negative mucosa was defined as WOS-negative

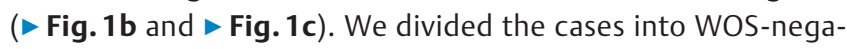
tive (WOS-0), for which WOS was negative at both the pyloric gland area and the fundic gland area, WOS-pyloric (WOS-P), for which WOS was positive at the pyloric gland area only, WOS-fundic (WOS-F), for which WOS was positive at the fundic gland area only, and WOS-pyloric plus fundic (WOS-P+F), for which WOS was positive at both the pyloric gland area and the fundic gland area. 


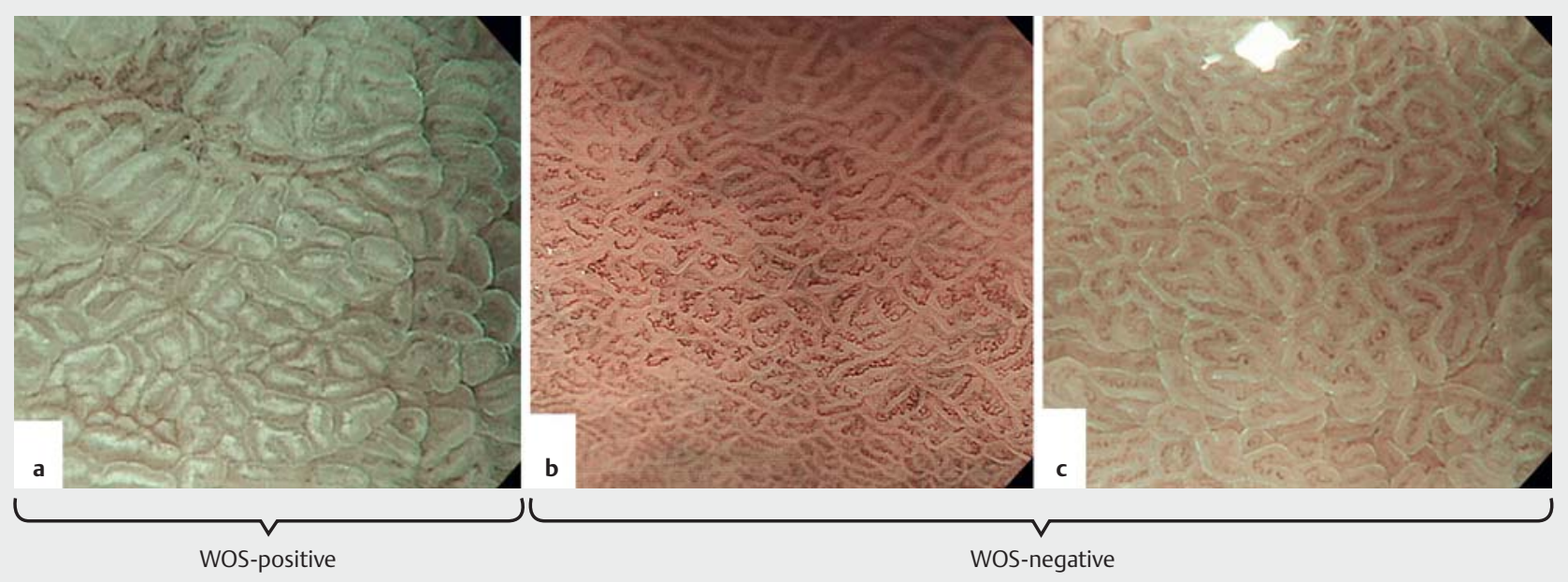

- Fig. 1 Definition of WOS-positive or WOS-negative. a WOS was defined as positive when a white substance was observed by M-NBI that obscured the subepithelial capillaries of the intervening part between crypt openings in the region of interest, regardless of LBC coexistence. b Normal mucosa. c LBC positive but WOS-negative mucosa was defined as WOS-negative.

\section{Definitions of $H$. pylori status}

To detect $H$. pylori infection, the following tests were used: serum Ig G antibody against $H$. pylori, ${ }^{13} \mathrm{C}$-urea breath test (UBT), urine antibody and stool antigen against $H$. pylori, and histological examination. A positive result for any test was diagnosed as " $\mathrm{H}$. pylori-infected." A negative result for least 1 test, without endoscopic atrophy, was diagnosed as "H. pylori-uninfected." When a UBT yielded negative results with a history of eradication therapy, a diagnosis of " $\mathrm{H}$. pylori-eradicated" was given. Subjects with a negative result for at least 2 of the tests, with endoscopic atrophy and no previous history of $H$. pylori eradication suggestive of spontaneous eradication, were excluded from the study.

\section{Proton pump inhibitor administration in $29 \mathrm{H}$. pylori-eradicated cases with gastric epithelial neoplasia}

We assumed that WOS would not be highly sensitive as an endoscopic marker for IM in patients after $H$. pylori eradication, especially in cases with acid recovery. To clarify this, we investigated the relationship between the presence of WOS and the $\mathrm{pH}$ level of fasting gastric juice before and after proton pump inhibitor (PPI) administration in $29 \mathrm{H}$. pylori-eradicated cases with gastric epithelial neoplasia. Of these cases, 9 adenomas and 20 differentiated adenocarcinomas were detected at least 6 mo after successful $H$. pylori eradication, and these patients received endoscopic resection of neoplasia. For the treatment of gastric ulcer after endoscopic resection, we administered PPIs. In all 29 cases, we measured fasting gastric juice $\mathrm{pH}$ and observed for WOS before and after PPI administration (mean $21 \mathrm{~d}$ ) according to the aforementioned procedure.

\section{End points}

The end points of the study were (1) the prevalence of WOS and the relationship between the presence of WOS and fasting gastric juice $\mathrm{pH}$ level in 3 groups $(H$. pylori-uninfected, $H$. pylori-infected, and $H$. pylori-eradicated); (2) the relationship between the presence of WOS and fasting gastric juice $\mathrm{pH}$ level before and after PPI administration in $29 \mathrm{H}$. pylori-eradicated cases.

\section{Statistical analysis}

Continuous variables were expressed as mean \pm standard deviation (SD) or $95 \%$ confidence interval $(\mathrm{Cl})$. For parametric variables, Student's t-test was used to compare the means between 2 groups; otherwise, a Wilcoxon's rank-sum test was used to compare the means of the groups. The chi-squared test or Fisher's exact test was used for comparisons of prevalence among the groups. McNemar's test was used to compare the prevalence of WOS before and after PPI administration in the $29 \mathrm{H}$. pylori-eradicated cases. A 2-sided p-value of less than 0.05 was considered statistically significant. Statistical analyses were performed using JMP-9 software (SAS Institute, Cary, NC).

\section{Results \\ Clinical features of patients divided into 3 groups by H. pylori status}

- Table 1 shows the characteristics of patients by dividing into 3 groups by $H$-pylori status ( $H$. pylori-uninfected, $H$. pylori-infected, and $H$. pylori-eradicated). Among 501 patients included in this study, the mean \pm SD age of the patients was $57.2 \pm 13.4 \mathrm{y}$. The male to female ratio was 303:198. Of the 501 patients, 206 (41.1\%) were $H$. pylori-uninfected, 109 (21.8\%) were H. pyloriinfected, and 189 (37.1\%) were H. pylori-eradicated. The mean period (mo) after $H$. pylori eradication in subjects was 36 mo (range: $7-180 \mathrm{mo}$ ). 
- Table 1 Clinical characteristics of patients divided into 3 H. pylori status.

\begin{tabular}{|c|c|c|c|c|}
\hline & $\begin{array}{l}\text { H. pylori-uninfected } \\
(\mathrm{n}=206)\end{array}$ & $\begin{array}{l}\text { H. pylori-infected } \\
(\mathrm{n}=109)\end{array}$ & $\begin{array}{l}\text { H. pylori-eradicated } \\
(\mathrm{n}=186)\end{array}$ & Total $(n=501)$ \\
\hline Mean age $\pm S D(y)$ & $49.0 \pm 10.5$ & $62.0 \pm 13.1$ & $64.4 \pm 11.7$ & $57.2 \pm 13.4$ \\
\hline Male/female, $\mathrm{n}$ & $109 / 97$ & $74 / 35$ & $120 / 66$ & $303 / 198$ \\
\hline $\begin{array}{l}\text { Gastric mucosal atrophy none/positive atrophy } \\
\text { (closed or open type) }\end{array}$ & $205 / 1$ & $4 / 105$ & $15 / 171$ & $224 / 277$ \\
\hline - Closed type atrophy, n (\%) & $1(0.5)$ & $30(27.5)$ & $96(51.6)$ & $127(25.3)$ \\
\hline - Open type atrophy, n (\%) & $0(0)$ & $75(68.8)$ & $75(40.3)$ & $150(30.0)$ \\
\hline Current or previous history of gastric tumor, $\mathrm{n}(\%)$ & $0(0)$ & $17(15.6)$ & $52(27.9)$ & $69(13.7)$ \\
\hline
\end{tabular}

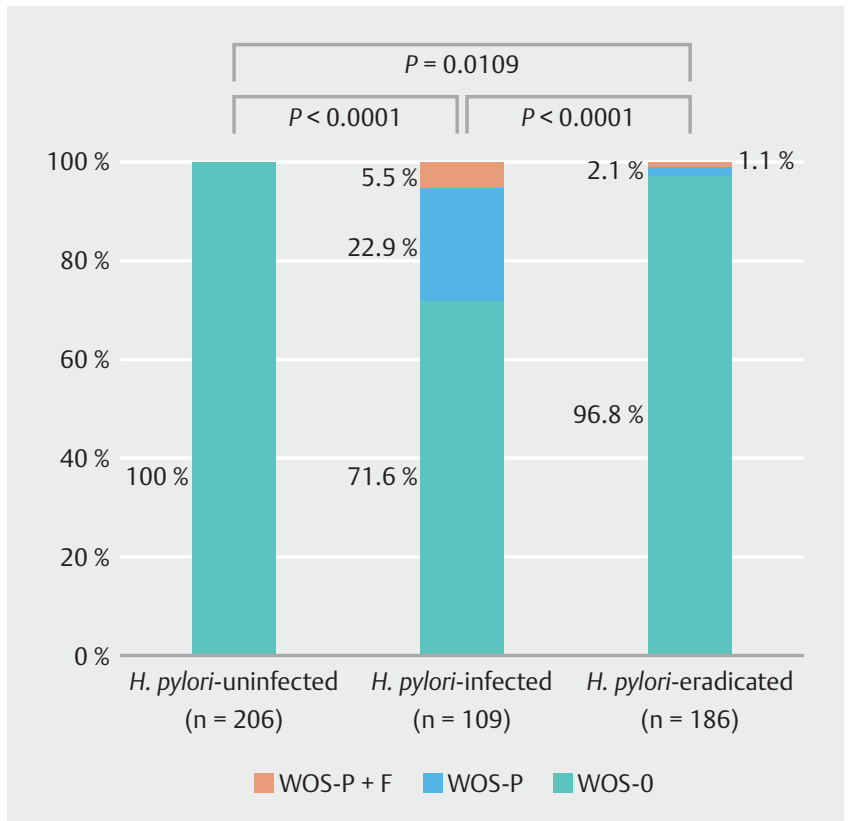

- Fig. 2 Prevalence of WOS in H. pylori-uninfected, H. pylori-infected, and $H$. pylori-eradicated groups.

According to the Kimura-Takemoto classification system for degree of gastritis [18], 224 (44.7\%) had no atrophy, 127 (25.3\%) had closed-type atrophic gastritis, and 150 (30.0\%) had open-type atrophic gastritis. Between the $H$. pylori-infected group and the $H$. pylori-eradicated group, there was no significant difference in the presence of atrophy $(P=0.2179)$. The presence of atrophy was $96.3 \%$ (closed type: $27.5 \%+$ open type: $68.8 \%$ ) in the $H$. pylori-infected group and was $91.9 \%$ (closed type: $51.6 \%+$ open type: $40.3 \%$ ) in the $H$. pylori-eradicated group.

Sixty-nine (13.7\%) patients had a history of treatment of gastric epithelial neoplasia. Between the $H$. pylori-infected group and the $H$. pylori-eradicated group, history of treatment of gastric epithelial neoplasia was significantly higher in the H. pylori-eradicated group (27.9\%) compared to the H. pylori-infected group (15.6\%) $(P=0.0158)$.
The prevalence of WOS in $\mathrm{H}$. pylori-uninfected, $H$. pylori-infected, and $H$. pylori-eradicated groups

Prevalence of WOS was $0 \%(0 / 206)$ in the $H$. pylori-uninfected group, $28.4 \%(31 / 109)$ in the $H$. pylori-infected group, and $3.2 \%(6 / 186)$ in the $H$. pylori-eradicated group ( $\mathbf{F i g} .2)$. A statistically significant difference in the prevalence of WOS was observed between the $H$. pylori-uninfected and $H$. pylori-infected groups $(P<0.0001)$ between the $H$. pylori-infected and $H$. pylori-eradicated groups $(P<0.0001)$ and between the $H$. pyloriuninfected and $H$. pylori-eradicated groups $(P=0.0109)$. The prevalence of WOS-P and WOS-P+F was $22.9 \%$ and $5.5 \%$, respectively, in the $H$. pylori-infected group and $2.1 \%$ and $1.1 \%$, respectively, in the $H$. pylori-eradicated group. There were no WOS-F cases.

Relationship between the presence of WOS and $\mathrm{pH}$ level of fasting gastric juice in $\mathrm{H}$. pylori-uninfected, $H$. pylori-infected, and $H$. pylori-eradicated groups

In the $H$. pylori-uninfected group, all cases were WOS-negative and the fasting gastric juice $\mathrm{pH}$ levels showed strong acidity (mean $\mathrm{pH}: 1.1,95 \% \mathrm{Cl}: 1.0-1.1)$. In the $H$. pylori-infected group, mean $\mathrm{pH}$ level $(95 \% \mathrm{Cl})$ of fasting gastric juice in the WOS-positive cases was significantly higher than that of the in WOS-negative cases (mean pH: 7.4 [7.1 - 7.6] vs. 4.6 [3.9-5.2], $P<0.0001)$. Similar to the $H$. pylori-infected group, the mean $\mathrm{pH}$ level of fasting gastric juice in the $H$. pylori-eradicated group was also significantly higher in WOS-positive cases than WOSnegative cases (mean $\mathrm{pH}: 7.2$ [6.1-8.2] vs. 1.6 [1.4-1.8], $P<$ $0.0001)$. We observed a few WOS-positive cases in the $H$. pylori-eradicated group, comprising $3.2 \%(n=6)$ of the total, and all of these fasting gastric juices demonstrated a neutral $\mathrm{pH}$ level (mean pH: 7.2, range: $6-8$ ) (\ Fig. 3 ).

\section{Relationship between the presence of WOS and $\mathrm{pH}$ level of fasting gastric juice before and after PPI administration in $29 \mathrm{H}$. pylori-eradicated cases with gastric epithelial neoplasia}

We investigated the relationship between the presence of WOS and fasting gastric juice $\mathrm{pH}$ before and after PPI administration in $29 \mathrm{H}$. pylori-eradicated cases with gastric tumor to clarify that presence of WOS may not be a highly sensitive endoscopic 

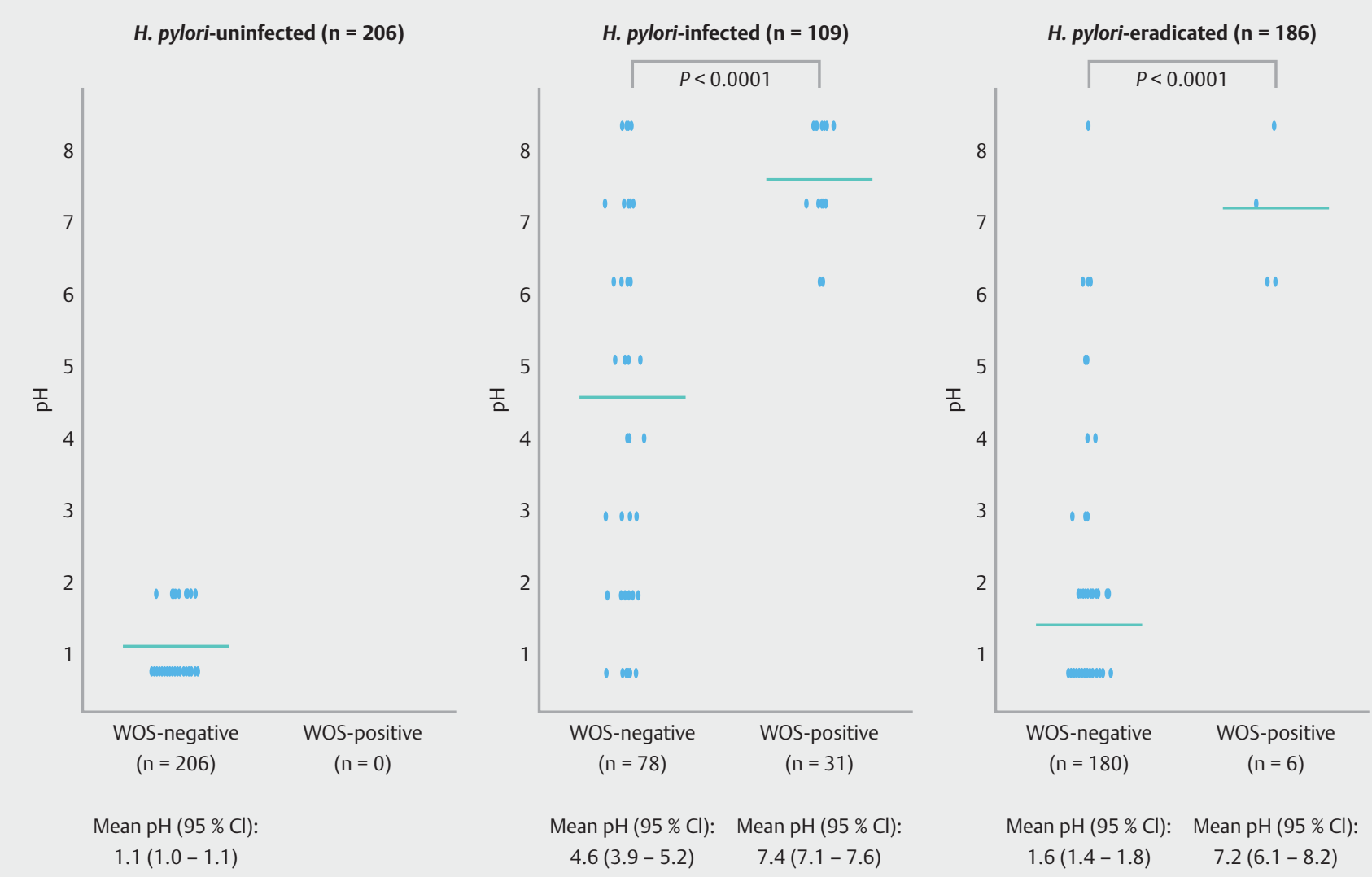

- Fig. 3 Relationship between the presence of WOS and fasting gastric juice pH level in H. pylori-uninfected, H. pylori-infected, and H. pylorieradicated groups.

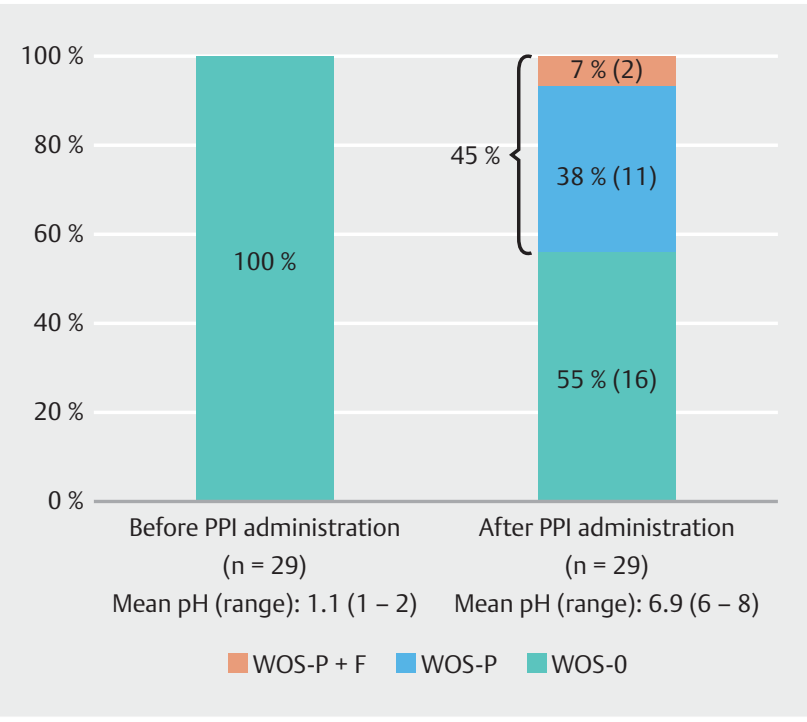

Fig. 4 Relationship between the presence of WOS and fasting gastric juice $\mathrm{pH}$ before and after PPI administration in $29 \mathrm{H}$. pylorieradicated cases with gastric epithelial neoplasia. marker of IM in patients after $H$. pylori eradication, especially in cases with acid recovery. The mean fasting gastric juice $\mathrm{pH}$ level in these 29 cases increased from 1.1 before PPI administration to 6.9 after. WOS positivity increased from $0 \%(0 / 29)$ before PPI

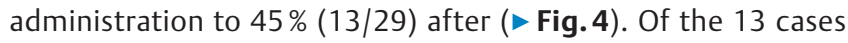
in which WOS appeared after PPI administration, the prevalence of WOS-P and WOS-P $+F$ was $11 / 13$ and $2 / 13$, respectively ( $\triangleright$ Fig. 4 and $\triangleright$ Fig.5). These findings suggested that the presence of WOS is not highly sensitive as an endoscopic marker for IM in patients after $H$. pylori eradication, especially in patients with acid recovery.

\section{Discussion}

In this study, we clarified the clinical significance of WOS as an optical marker for IM and its limitations in the changing gastric acid conditions in various $H$. pylori states. The presence of WOS in IM was thought to be closely associated with a hypochlorhydria state in atrophic gastric mucosa caused by $\mathrm{H}$. pylori infection. However, WOS may not be highly sensitive as an endoscopic marker for IM in patients with acid recovery after $H$. pylori eradication.

The study results demonstrated that the presence of WOS in IM was closely associated with a hypochlorhydria state caused by $H$. pylori infection. WOS, as observed by M-NBI, was most fre- 


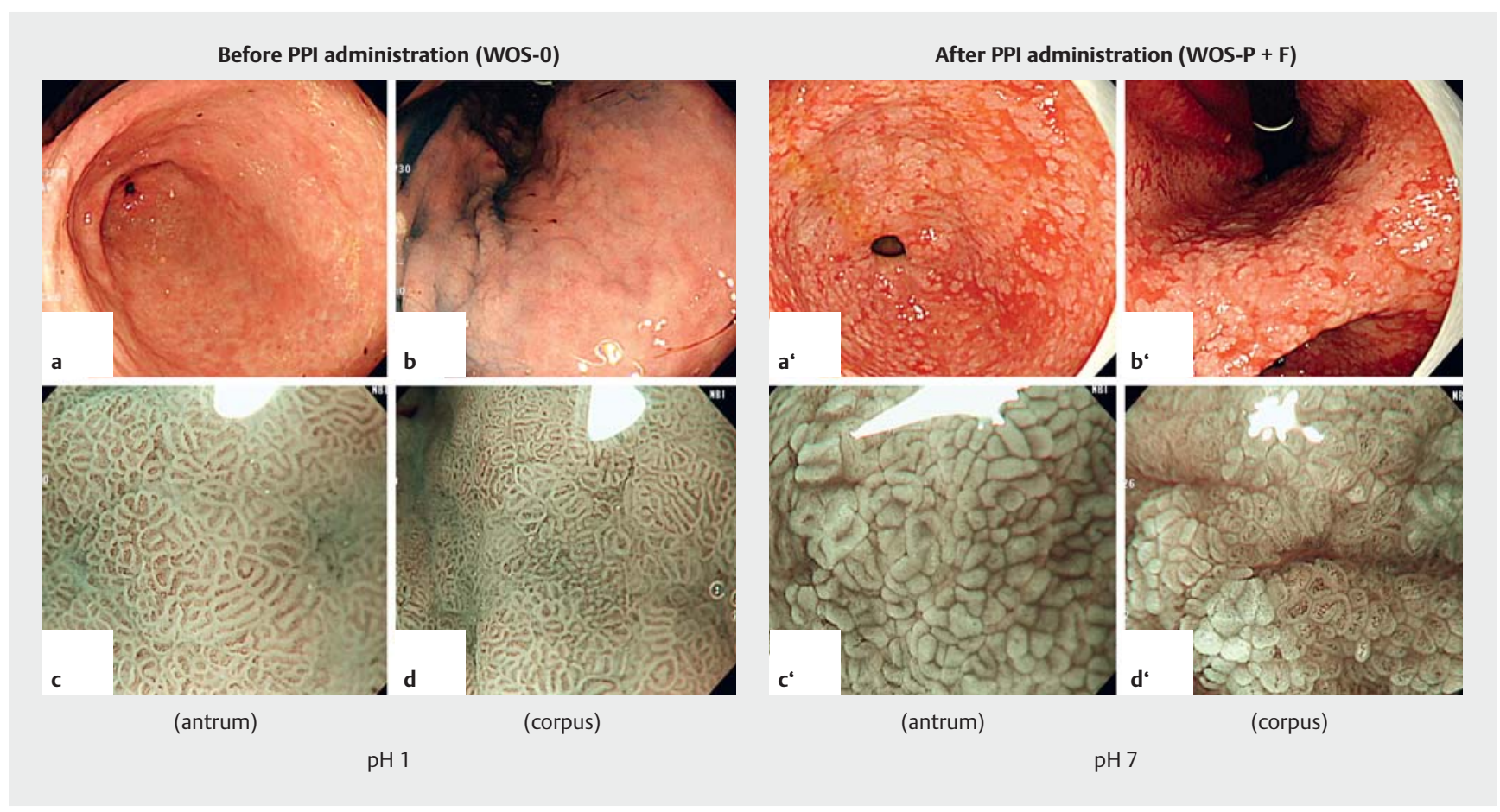

- Fig. 5 A representative $H$. pylori-eradicated case with WOS appearance: endoscopic findings before and after PPI administration. WOS is not observed before PPI administration (a-d). However, WOS appears dramatically after $14 \mathrm{~d} \mathrm{PPI}$ administration (a' - d'). Fasting gastric juice pH changed from $\mathrm{pH} 1$ to $\mathrm{pH} 7$ following $\mathrm{PPI}$ administration.

quently found in the $H$. pylori-infected group $(28.4 \%)$, infrequently observed in $\mathrm{H}$. pylori-eradicated cases (3.2\%), and not found at all in $\mathrm{H}$. pylori-uninfected cases. In addition, there was a close relationship between the presence of WOS and fasting gastric juice pH level. Among the $295 \mathrm{H}$. pylori-infected and H. pylori-eradicated cases, fasting gastric juice $\mathrm{pH}$ level was significantly higher in WOS-positive cases (mean pH 7.4 and 7.2, respectively) than in WOS-negative cases (mean $\mathrm{pH} 4.6$ and 1.6 , respectively). Interestingly, All of WOS-positive cases in the $\mathrm{H}$. pylori-eradicated group ( $3.2 \%$ of the total) showed neutral fasting gastric juice pH levels (mean $\mathrm{pH} 7.2$, range 6-8), even after successful $H$. pylori eradication. From these findings, we concluded that a WOS-positive state was closely associated with neutralization of intragastric $\mathrm{pH}$ in $\mathrm{H}$. pylori-infected and $\mathrm{H}$. pylori-eradicated cases.

As the degree of mucosal atrophy progresses, acidity in the stomach is neutralized to a pH level of approximately 7.0 [19]. In contrast, $H$. pylori eradication therapy contributes to the normalization of gastric juice $\mathrm{pH}$ owing to a recovery of acid secretion, not only in the intact stomach but also in the remaining stomach after gastrectomy $[20,21]$. However, some patients with profound hypochlorhydria may not recover because of progression to an irreversible stage [22]. It is well known that chronic gastric acid hypochlorhydria and achlorhydria are important risk factors for the development of gastric cancer [23, 24]. Therefore, endoscopic investigations that allow speculation of gastric acid conditions are highly desirable. The mechanism of WOS expression suggests that presence of WOS could be a marker representing neutralization of intragastric $\mathrm{pH}$ due to $H$. pylori infection. Accordingly, WOS might be an important marker for predicting high risk for the development of gastric cancer.

Furthermore, the study results demonstrate the limitation of WOS as a marker for IM. That is, WOS might not be highly sensitive as an endoscopic marker for IM in patients after $H$. pylori eradication with acid recovery. Between the $H$. pylori-infected group and the $H$. pylori-eradicated group, there was no significant difference in the presence of atrophy by Kimura-Takemoto classification system $(P=0.2179)$. The presence of atrophy was $96.3 \%$ in the H. pylori-infected group and was $91.9 \%$ in the H. pylori-eradicated group. On the other hand, treatment history of gastric neoplasia was significantly higher in the $\mathrm{H}$. pylori-eradicated group (27.9\%) compared to the H. pylori-infected group $(15.6 \%)(P=0.0158)$. However, WOS was significantly lower ( $3.3 \%$ ) in the H. pylori-eradicated group compared to the H. pylori-infected group (28.4\%) in spite of there being no significant difference on the presence of atrophy representative of a surrogate marker of IM. In addition, we investigated the relationship between the presence of WOS and fasting gastric juice $\mathrm{pH}$ level before and after PPI administration in $29 \mathrm{H}$. pylori-eradicated cases with gastric epithelial neoplasias. Although we could not observe any WOS in these $\mathrm{H}$. pylori-eradicated cases with acid recovery, WOS dramatically appeared in $45 \%$ of these cases following neutralization of intragastric $\mathrm{pH}$ with PPI administration. From these findings, we concluded that the presence of WOS is not a consistent phenomenon. Therefore, in our routine screening endoscopy, we should pay attention for the evaluation of IM by WOS findings if a patient has a history of $\mathrm{H}$. pylori eradica- 
tion. If WOS is not detected, the existence of histological IM cannot be completely excluded. If WOS is detected in patients even after $H$. pylori eradication, not only is the existence of histologic IM suggested, but also that acid secretion has not yet recovered. In other words, such patients may have a high risk for developing gastric cancer despite the eradication of $H$. pylori, since chronic gastric acid hypochlorhydria and achlorhydria are important risk factors for the development of gastric cancer $[23,24]$.

Kanemitsu et al., [7] recently demonstrated that the presence of WOS, as observed by $\mathrm{M}-\mathrm{NBI}$ as a novel endoscopic parameter for the histological diagnosis of IM, had a sensitivity and specificity of $50 \%$ and $100 \%$, respectively. However, in patients with $\mathrm{H}$. pylori eradication, diagnosing IM using WOS findings alone may not be sufficiently sensitive. Our results suggested that WOS was not highly sensitive despite histological IM because WOS is affected by some of the intragastric environment. Ohtsu et al. [25] revealed that the oral ingestion of foods containing fats increased the prevalence of WOS in gastric epithelial neoplasia. We believe that the intragastric $\mathrm{pH}$ environment is also an important factor, with the activation of lipase, including pancreatic, lingual and gastric types, increasing the prevalence of WOS. It may be necessary to clarify how the combination of antacid and emulsified fat foods contribute to the emergence of WOS.

The study had several limitations. First, it was retrospective and conducted at a single center. Second, gastric acid conditions were only evaluated by fasting gastric juice $\mathrm{pH}$ level. Although $\mathrm{pH}$ measurement of fasting gastric juice is one of reliable information for gastric acid condition, it is not an ideal method for evaluation of acid secretion itself. Third, we did not study the relationship of other optical markers such as LBC with histological IM, regardless of $H$. pylori eradication status. Finally, the patients included in this study received endoscopy for mainly screening purposes, so we could not examine IM histologically. A further well-designed prospective study with histologic examination is expected to clarify these issues.

In conclusion, the results of this study suggest that the presence of WOS in $\mathrm{H}$. pylori-infected gastritis is closely associated with a neutralization of intragastric $\mathrm{pH}$ due to $\mathrm{H}$. pylori infection but is not a highly sensitive endoscopic marker of IM in patients after $H$. pylori eradication.

\section{Acknowledgements}

This work was supported by the Central Research Institute for Endoscopy, Fukuoka University.

Competing interests

None

\section{References}

[1] Uemura N, Okamoto S, Yamamoto S et al. Helicobacter pylori infection and the development of gastric cancer. N Engl J Med 2001; 345: 784 789

[2] Shichijo S, Hirata Y, Sakitani K et al. Distribution of intestinal metaplasia as a predictor of gastric cancer development. J Gastroenterol Hepatol 2015; 30: 1260 - 1264

[3] Correa P. Chronic gastritis as a cancer precursor. Scand J Gastroenterol Suppl 1984; 104: 131-136

[4] De Vries AC, van Grieken NC, Looman CW et al. Gastric cancer risk in patients with premalignant gastric lesions: a nationwide cohort study in the Netherlands. Gastroenterology 2008; 134: 945 - 952

[5] Sugano K, Tack J, Kuipers E] et al. Kyoto global consensus report on Helicobacter pylori gastritis. Gut 2015; 64: 1353 - 1367

[6] Uedo $\mathrm{N}$, Ishihara $\mathrm{R}$, lishi $\mathrm{H}$ et al. A new method of diagnosing gastric intestinal metaplasia: narrow-band imaging with magnifying endoscopy. Endoscopy 2006; 38: 819-824

[7] Kanemitsu T, Yao K, Nagahama T et al. Extending magnifying NBI diagnosis of intestinal metaplasia in the stomach: the white opaque substance marker. Endoscopy 2017; 49: 529-535

[8] Yao K, Iwashita A, Tanabe $\mathrm{H}$ et al. White opaque substance within superficial elevated gastric neoplasia as visualized by magnification endoscopy with narrow-band imaging: a new optical sign for differentiating between adenoma and carcinoma. Gastrointest Endosc 2008; 68: $574-580$

[9] Yao K, Iwashita A, Nambu M et al. Nature of white opaque substance in gastric epithelial neoplasia as visualized by magnifying endoscopy with narrow-band imaging. Dig Endosc 2012; 24: 419-425

[10] Ueo T, Yonemasu H, Yada $\mathrm{N}$ et al. White opaque substance represents an intracytoplasmic accumulation of lipid droplets: immunohistochemical and immunoelectron microscopic investigation of 26 cases. Dig Endosc 2013; 25: 147 - 155

[11] Rubin W, Ross LL, Jeffries GH et al. Some physiologic properties of heterotopic intestinal epithelium. Its role in transporting lipid into the gastric mucosa. Lab Invest 1967; 16: 813-827

[12] Siurala M, Tarpila S. Absorptive function of intestinal metaplasia of the stomach. Scand J Gastroenterol 1968; 3: 76 - 79

[13] Borgstrom B, Brockman HL. Lipases. Amsterdam: Elsevier; 1984

[14] Chen HN, Wang Z, Li X et al. Helicobacter pylori eradication cannot reduce the risk of gastric cancer in patients with intestinal metaplasia and dysplasia: evidence from a meta-analysis. Gastric Cancer 2016; 19: $166-175$

[15] Ueo T, Yanai Y, Ishida T. [Presence of hematin represents lower intragastric pH level]. Gastroenterol Endosc 2015; 57 : 2054

[16] Segawa K, Nakazawa S, Tsukamoto Y et al. Estimate of gastric acid output by evaluation of fasting gastric juice collected endoscopically. Hepatogastroenterology 1991; 38: 79-82

[17] Fukuta N, Ida K, Kato T et al. Endoscopic diagnosis of gastric intestinal metaplasia: a prospective multicenter study. Dig Endosc 2013; 25: $526-534$

[18] Kimura K, Takemoto T. An endoscopic recognition of the atrophic border and its significance in chronic gastritis. Endoscopy 1969; 3: $87-97$

[19] Feldman M, Cryer B, Sammer D et al. Influence of H. pylori infection on meal-stimulated gastric acid secretion and gastroesophageal acid reflux. Am J Physiol 1999; 277: 1159-1164

[20] Taguchi Y, Kaito M, Gabazza EC et al. Helicobacter pylori inhibits the secretory activity of gastric parietal cells in patients with chronic gastritis. An ultrastructural study. Scand J Gastroenterol 1997; 32: 656 663 
[21] Kato S, Matsukura N, Matsuda N et al. Normalization of pH level and gastric mucosa after eradication of $H$. pylori in the remnant stomach. J Gastroenterol Hepatol 2008; 23: 258-261

[22] lijima K, Sekine H, Koike T et al. Long-term effect of Helicobacter pylori eradication on the reversibility of acid secretion in profound hypochlorhydria. Aliment Pharmacol Ther 2004; 19: 1181-1188

[23] Svendsen JH, Dahl C, Svendsen LB et al. Gastric cancer risk in achlorhydric patients. A long-term follow-up study. Scand J Gastroenterol 1986; $21: 16-20$
[24] Argent RH, Thomas RJ, Aviles-Jimenez F et al. Toxigenic Helicobacter pylori infection precedes gastric hypochrorhydria in cancer relatives, and $H$. pylori virulence evolves in these families. Clin Cancer Res 2008; 14: $2227-2235$

[25] Ohtsu K, Yao K, Matsunaga K et al. Lipid is absorbed in the stomach by epithelial neoplasms (adenomas and early cancers): a novel functional endoscopy technique. Endosc Int Open 2015; 3: E318 - E322 